\title{
Nicolas Balzamo, Les deux cathédrales
}

Mythe et histoire à Chartres (XIe-XXe siècle)

\section{Florian Mazel}

\section{(2) OpenEdition Journals}

Édition électronique

URL : http://journals.openedition.org/abpo/2911

DOI : $10.4000 / a b p o .2911$

ISBN : 978-2-7535-3977-8

ISSN : 2108-6443

Éditeur

Presses universitaires de Rennes

\section{Édition imprimée}

Date de publication : 15 décembre 2014

Pagination : 195-199

ISBN : 978-2-7535-3975-4

ISSN : 0399-0826

\section{Référence électronique}

Florian Mazel, « Nicolas Balzamo, Les deux cathédrales », Annales de Bretagne et des Pays de l'Ouest [En ligne], 121-4 | 2014, mis en ligne le 15 décembre 2014, consulté le 23 septembre 2020. URL : http:// journals.openedition.org/abpo/2911; DOI : https://doi.org/10.4000/abpo.2911 
çais. Ces rénovations ont souvent été l'occasion de développer une approche mémorielle et patrimoniale de ces quartiers trop systématiquement décriés. Il serait heureux qu'ils servent de points de départ à une histoire plus générale de ces territoires spatialement et symboliquement considérables de la France urbaine contemporaine.

Gwenaëlle LEGOULLON

BaLZAMO, Nicolas, Les deux cathédrales. Mythe et histoire à Chartres (XI'-XXe siècle), Paris, Les Belles Lettres, 2012, 382 p.

Si la cathédrale de Chartres est universellement reconnue comme l'un des joyaux de l'architecture gothique ${ }^{1}$, la légende chartraine, cette seconde cathédrale évoquée par le titre du présent ouvrage, est aujourd'hui assez largement tombée dans l'oubli en dépit de son ampleur. C'est à l'étude dans la longue durée de cette légende, que Nicolas Balzamo a choisi de considérer comme un véritable "mythe de fondation " de l'Église de Chartres, qu'est consacré ce stimulant ouvrage. En bon herméneute des récits de fondation médiévaux et modernes, lesquels font aujourd'hui l'objet de recherches particulièrement dynamiques illustrées notamment par les maîtreslivres d'Amy Remensnyder et Roberto Bizzochi ${ }^{2}$, Nicolas Balzamo ne cherche pas à démêler le vrai du faux. Il ne part pas non plus en quête d'une d'improbable origine, mais prend acte d'un système de croyance, ou plutôt d'un régime de vérité qui a nourri jusqu'au milieu du XVII e siècle l'adhésion unanime des chrétiens d'autrefois et a même connu, après une première phase de doute critique de près de deux siècles, un surprenant revival dans la France catholique de la deuxième moitié du XIX ${ }^{\mathrm{e}}$ siècle et du début du $\mathrm{xx}^{\mathrm{e}}$ siècle. Pour cela, l'auteur s'efforce de recomposer, par strates successives, le processus de constitution et de transmission du mythe en suivant le fil rouge des objets et des récits.

L'originalité de la légende chartraine, au regard des nombreuses traditions liées aux fondations monastiques ou aux origines des sièges épiscopaux, réside dans la connaissance prophétique que les druides du pays des Carnutes auraient eu de la révélation chrétienne bien avant l'Incarnation, connaissance manifestée par la sculpture d'une mystérieuse statue de bois de la "Vierge devant enfanter ». Cette légende faisait de l'église de Chartres le plus ancien sanctuaire chrétien consacré à la Vierge puisqu'il était antérieur à l'existence même de Marie et à la fondation du siège épiscopal, plus classiquement attribué à des disciples de l'apôtre Pierre venus de la métropole de Sens - la légende enracinait ainsi la hiérarchie des sièges aux origines du christianisme -, Savinien, Potentien et Altin, les premiers étant considérés comme deux des 72 disciples mentionnés dans les Écritures. On ne s'étonnera pas, dans ces conditions, que la fabrique légendaire alla jusqu'à engendrer la production d'une lettre aux Chartrains attribuée à la Vierge elle-même.

Comme le montre l'auteur, une autre des forces de la légende chartraine réside dans l'utilisation de plusieurs objets matériels associés au culte. Le premier de ces objets est bien sûr la relique de la Vierge, un " morceau de soie blanche écrue, sans

1. Signalons la parution récente du volume consacré à Chartres dans la collection " La grâce d'une cathédrale ", auquel a d'ailleurs participé Nicolas Balzamo (Chartres, Strasbourg, 2013).

2. REMENSNYDER, Amy Goodrich, Remembering Kings Past. Monastic Foundation Legends in Medieval Southern France, Ithaca, 1995; Bizzocchi, R., Généalogies fabuleuses. Inventer et faire croire dans l'Europe moderne, Paris, 2010 (1 $1^{\text {re }}$ éd. italienne Bologne, 1995). 
broderie ni coutures", connu sous le nom de chemise (camisa) de la Vierge, mais dont la nature exacte fit l'objet d'appréciations diverses au cours de l'histoire (chemise, voile, tunique). Mentionnée dans un récit miraculeux du siège de la cité par les Normands en 911 de l'Histoire des ducs de Normandie de Dudon de Saint-Quentin, un texte sans rapport avec le milieu chartrain composé au début du XI ${ }^{\mathrm{e}}$ siècle, sa présence dans la cathédrale n'est véritablement assurée, grâce au nécrologe du chapitre, qu'à partir du milieu du $x^{\mathrm{e}}$ siècle (son offrande par Charles le Chauve n'est en revanche qu'une hypothèse). Cette relique a elle-même suscité sa propre légende parallèle dont les premiers éléments remontent à Constantinople à la fin du $\mathrm{V}^{\mathrm{e}}$ siècle. La légende fut ensuite développée par les chroniqueurs normands, anglais et dyonisiens des $\mathrm{Xl}^{\mathrm{e}}$-xII ${ }^{\mathrm{e}}$ siècles, bien avant d'être appropriée par le milieu chartrain à partir du milieu du $\mathrm{XII}^{\mathrm{e}}$ siècle (ajout à la chronique de Saint-Père) et plus encore au XIII siècle (Livre des miracles). Le deuxième objet n'est autre que la statue de la "Vierge devant enfanter ", dont la présence est attestée dans la crypte de la cathédrale à partir du XIV ${ }^{\mathrm{e}}$ siècle et qui y fut peut-être exposée dès le XII ${ }^{\mathrm{e}}$ siècle. Enfin, un dernier " objet " est constitué par le puits des Saints-Forts, dans la crypte de la cathédrale, réputé pour être le lieu du martyre des premiers évangélisateurs de la cité.

Au regard de l'originalité de son objet d'étude, la démarche de Nicolas Balzamo s'avère elle-même doublement originale. Une première originalité tient au choix de la longue durée, qui conduit l'auteur à remonter le plus loin possible (en l'occurrence jusqu'au ve siècle, mais bien loin de Chartres) et à prolonger l'enquête jusqu'au $\mathrm{XX}^{\mathrm{e}}$ siècle (même si le chapitre consacré à l'époque contemporaine est à vrai dire très rapide), alors que la plupart des historiens se concentrent soit sur la séquence médiévale, soit sur la période moderne, au gré de leur compétence ou de leur centre d'intérêt. Cette histoire de longue haleine, qui fait feu de tout bois (récits, objets, images), fonde le plan de l'ouvrage, dont les trois parties correspondent de manière assez classique aux trois temps de fabrique et de réception de la légende. La première partie, intitulée " Genèse ", met en relief le rôle de la relique de la Vierge, vecteur d'un pèlerinage dont le succès est souligné par de nombreux chroniqueurs du XII ${ }^{\mathrm{e}}$ siècle du nord de la France et qui semble dynamisé par les campagnes de reconstruction de la cathédrale (1130-1140 et 1194-1220) et la rédaction de deux livres de miracles, l'un en latin au tout début du XIII ${ }^{\mathrm{e}}$ siècle, l'autre en français, dû à Jean le Marchant, vers 1250-1260. Ce moment est surtout celui de la captation finalement tardive (peu avant 1130) par le milieu chartrain de motifs apparus ailleurs, à l'abbaye Saint-Pierre-le-Vif de Sens pour les légendes d'évangélisation (la Grande Passion de l'abbé Gerbert) et chez les chroniqueurs anglo-normands pour le miracle de la chemise (Dudon, Guillaume de Malmesbury, Robert de Torigny...).

La deuxième partie, intitulée " Affirmation ", décrit la cristallisation des différentes légendes et la formulation progressive d'un récit synthétique depuis la chronique des évêques (1389) jusqu'aux grandes histoires des $\mathrm{XVI}^{\mathrm{e}}$ et XVII ${ }^{\mathrm{e}}$ siècles. C'est également le moment où le dispositif liturgique du sanctuaire apparaît le plus abouti, associant la statue, la relique et le puits des Saints-Forts. C'est enfin le moment de la réception la plus large du mythe au-delà des cercles ecclésiastiques et des troupes pèlerines, auprès des élites urbaines, qui l'utilisent dans les rituels publics, se regroupent en confrérie de la Vierge (1506) et l'instrumentent contre les protestants lors des guerres de religion; auprès des rois de France, de Jean le Bon à Louis XIII; auprès de la papauté, le préambule de la bulle du $1^{\text {er }}$ janvier 1517 labellisant en quelque sorte la légende locale. Il n'est pas jusqu'aux milieux érudits (Belleforest en 1575, Claude Duparc en 1558, Sébastien Rouillard en 1609, Claude Savard en 1671) qui n'en confortent les principaux éléments dans le cadre du nouveau discours humaniste. 
La troisième partie, intitulée " Déclin ", évoque la décomposition progressive de l'édifice légendaire. Les premiers coups viennent de l'érudition ecclésiastique, d'abord de la plume même de chanoines de Chartres tels Jean-Baptiste Souchet (1654), ce "Mabillon de province ", ou Léger-François Brillon, puis des bénédictins de la Gallia christiana (1744), autant d'œuvres qui correspondent par ailleurs de plus en plus aux aspirations des évêques en faveur d'une "épuration " historiographique et liturgique (le bréviaire est expurgé des pseudo-saints fondateurs) de légendes désormais tenues pour populaires et superstitieuses. En détruisant les principaux signes matériels du culte (la statue de bois, la chemise de la Vierge, dont seul un lambeau survécut), la Révolution achève une évolution plus qu'elle ne commet un véritable sacrilège. Le " mythe chartrain " connut cependant un étonnant chant du cygne avec la restauration catholique du second $\mathrm{XIX}^{\mathrm{e}}$ siècle. Paradoxalement, ce fut le moment où le pèlerinage de Chartres acquit pour la première fois une véritable dimension nationale dans un contexte de mariolâtrie exacerbée et d'expiation patriotique (dogme de l'Immaculée conception, apparitions de Lourdes, choix de Chartres pour le pèlerinage pénitentiel de $1873 . .$.$) vigoureusement entretenues$ par les prélats locaux. Cette renaissance s'essouffla dès l'entre-deux-guerres, mais elle fut suffisamment intimidante pour peser sur les premiers travaux de l'historiographie positiviste locale (Eugène de Lépinois et Lucien Merlet), au grand dam de $\mathrm{M}^{\mathrm{gr}}$ Duchesne, pourfendeur de la «mythologie " chartraine. En trois tableaux, Nicolas Balzamo opère donc une stratigraphie complète des légendes chartraines, en se montrant à chaque fois soucieux d'insérer chaque nouveau récit et chaque innovation liturgique dans son contexte de production et dans le système social et mental qui le justifie.

Car la deuxième originalité de la démarche de Nicolas Balzamo réside, au-delà de cette stratigraphie, dans une approche globale qui resitue la légende chartraine dans le cadre du " grand légendier chrétien " (Alain Boureau ${ }^{3}$ ), dans la veine des nombreux travaux soulignant l'étroite articulation entre la " grande " histoire sainte universelle (celle des Écritures) et la multiplicité des petites histoires saintes locales. C'est pour mieux fonder cette approche globale que Nicolas Balzamo a choisi de recourir à la notion de mythe et plus particulièrement à celle de mythe de création ou de fondation, plutôt qu'aux notions plus familières aux historiens, surtout médiévistes, de légende ou de tradition ${ }^{4}$. Même s'il déclare l'avoir en partie fait par défaut, l'auteur s'explique à deux reprises sur ce choix singulier et audacieux : brièvement dans son introduction, plus longuement dans une vaste conclusion générale qui, comme son titre l'indique ("Le mythe : essai d'interprétation générale ") constitue en fait une sorte de synthèse. La notion de mythe présente il est vrai certains avantages : elle permet de considérer les légendes comme un corpus cohérent de croyances à la fois stratifiées (en fonction de la place de chacun des auteurs dans l'Église et la société) et incarnées dans des pratiques sociales, et non comme un tissu de fables naïves ou le fruit de manipulations intéressées; elle permet également d'affiner la compréhension des différents éléments ou caractères du mythe par le recours à un comparatisme mesuré (encore que cet avantage reste assez théorique, l'auteur ne se livrant finalement qu'à bien peu de comparaisons avec d'autres légendes ecclésiastiques); enfin et surtout la notion de mythe permet d'écarter les

3. Alain BOUREAU, «Le grand légendier chrétien ", dans id., L'événement sans fin. Récit et christianisme au Moyen Âge, Paris, 1993, p. 15-37.

4. Ceci explique la publication de l'ouvrage dans la collection "Vérité des mythes " des Belles Lettres plutôt que dans la collection " Histoire", ainsi que l'absence des études d'histoire spécialisées citées dans les notes dans la bibliographie générale, ce qui à vrai dire n'est guère commode. 
interprétations en termes de culture populaire (ou folklorique) ou de culture lettrée, le " mythe chartrain " (comme tous les mythes médiévaux à vrai dire) apparaissant clairement comme une " création savante élaborée par les clercs, pour les clercs et à l'aide de la culture écrite " (p. 221). En outre, l'auteur est bien conscient de la nécessité d'adapter la notion de mythe au contexte d'une société chrétienne : le "mythe chartrain ", comme tous les mythes locaux, s'insère en effet dans la " méta-mythologie " que constitue le christianisme (p. 216), dont la spécificité par rapport aux mythes traditionnels est l'historicité, c'est-à-dire l'intégration dans une histoire sainte linéaire exposée par les Écritures et prolongée par l'hagiographie. Le " mythe chartrain " est donc avant tout une généalogie de l'Église de Chartres et son effondrement, engagé à partir du milieu du XVII e siècle, prend place dans le vaste mouvement de " démythologisation " qui affecte tout le christianisme, ce qui rend d'autant plus spectaculaire, mais aussi très fragile, la tentative de résurrection du second XIX ${ }^{\mathrm{e}}$ siècle.

Cependant, en dépit de ces avantages et de ces précautions, la notion de mythe n'emporte pas vraiment la conviction, en particulier lorsqu'elle est sert à rejeter toute analyse fonctionnaliste de la légende, ce que l'auteur appelle son « introuvable utilité " (p. 244). Certes, il est juste de relativiser le lien entre la légende et le pèlerinage : dans la postérité des travaux d'André Chédeville ${ }^{5}$, l'auteur souligne à bon escient le faible rayonnement du pèlerinage, qui demeure (sauf au XIX $\mathrm{X}^{\mathrm{e}}$ siècle donc) à la fois très local et très élitiste - au-delà du pays chartrain ce sont les souverains et les princes qui multiplient les dévotions envers le sanctuaire chartrain - et ne rapporte pas grand-chose au chapitre au regard des revenus de la seigneurie. Certes, il est juste également de critiquer les études d'André Sanfaçon qui voyait dans le mythe une tentative du chapitre de compenser sur le terrain symbolique la perte de son pouvoir réel face à l'évêque (renforcé par la réforme tridentine), au roi et aux élites laïques ${ }^{6}$, car comme le montre l'auteur le mythe est pleinement constitué bien avant les XVI ${ }^{\mathrm{e}}$-XVII ${ }^{\mathrm{e}}$ siècles.

En revanche, il me semble impossible de comprendre la fabrique légendaire des $\mathrm{XII}^{\mathrm{e}}$-XIII ${ }^{\mathrm{e}}$ siècles sans la référer au processus d'affirmation sans précédent des églises diocésaines dans l'institution ecclésiale et dans la société chrétienne dans la postérité de la réforme grégorienne. À ce titre, il importe peu que la fabrique chartraine ne soit pas pleinement indigène mais découle largement de la captation de traditions apparues ou élaborées ailleurs, à Sens, en Normandie ou en Angleterre. Il n'y a pas lieu pour autant d'y voir le signe du " hasard " (p. 49 et 235), car cette captation intentionnelle s'accompagne d'une appropriation au bénéfice du chapitre cathédral qui représente alors l'institution appelée à incarner au mieux l'idéal de la réforme (comme figure du collège apostolique), la médiation sacerdotale (par le contrôle des paroisses et des dîmes) et l'identité de chaque église locale (par l'exaltation d'une généalogie $^{7}$, de lieux ${ }^{8}$ et d'objets sacrés spécifiques ${ }^{9}$ ). Le jeu de correspondances entre la fabrique légendaire et les chantiers de la cathédrale s'intègre parfaitement dans ce contexte qui fait de la "Maison Dieu ", de ses autels et de ses images, un instrument et non seulement le réceptacle d'un discours d'exaltation de la média-

5. CHÉDEVILLE, André, Chartres et ses campagnes (XI -XII ${ }^{e}$ s.), Paris, 1973, p. 505-519.

6. Notamment SANFAÇON, André, "Légendes, histoire et pouvoir à Chartres sous l'Ancien Régime ", Revue historique, 279, 1988, p. 337-357.

7. Ici l'événement miraculeux de 811, puis les origines byzantines (ce n'est qu'au XIV ${ }^{\mathrm{e}}$ siècle que le mythe druidique s'impose).

8. Ici la crypte de la cathédrale et le puits des Saints-Forts.

9. Ici la chemise et la statue de la Vierge. 
tion ecclésiale dont la Vierge-Marie-Mère-Église devient la figure emblématique ${ }^{10}$, permettant du même coup à l'évêque de s'associer aux entreprises idéelle et matérielle menées par le chapitre. Les $\mathrm{XII}^{\mathrm{e}}$-XIII ${ }^{\mathrm{e}}$ siècles constituent enfin le moment de la réaffirmation à la fois institutionnelle et seigneuriale des sièges épiscopaux et des chapitres cathédraux face aux communautés monastiques, dont les légendes de fondation avaient fleuri aux $\mathrm{x}^{\mathrm{e}}$-xII ${ }^{\mathrm{e}}$ siècles : dans cette compétition également l'appropriation du culte marial apparaît centrale, de même que l'assimilation des chanoines aux 72 disciples du Christ. En définitive, la genèse de la légende chartraine correspond au moment où s'affirme le dominium de l'institution ecclésiale en général et des églises cathédrales en particulier. À ce titre, les enjeux de l'apparition, sous la plume de Guibert de Nogent, de l'appellation "dame de Chartres ", qui devient peu à peu proverbiale - elle est notamment reprise par les recueils de miracles du XIII ${ }^{\mathrm{e}}$ siècle - ne me semblent pas vraiment perçus. Car ce que dissimule le terme " dame " (domina), aujourd'hui banalisé dans la langue française, c'est bien la référence à l'exercice d'un dominium qui n'est pas seulement revendication d'une seigneurie de la Vierge sur la cité de Chartres comme le souligne l'auteur à la suite de Jean le Marchant, ni simplement un écho de la culture courtoise sur le culte marial, mais l'énoncé explicite de la domination de l'institution ecclésiale, exprimée en terme à la fois féodal et seigneurial, sur l'ensemble de la société chrétienne, et dont l'Église de Chartres n'est en définitive qu'une figure. Dans ce contexte, la raison d'être de la fabrique légendaire réside bien dans sa puissance performative d'affirmation de l'autorité institutionnelle.

Je ne suis pas en capacité de suggérer une lecture identique des autres séquences du " mythe chartrain " mises en relief par Nicolas Balzamo, à commencer par ce moment de déconstruction de l'intérieur du milieu du XVII ${ }^{\mathrm{e}}$ siècle. Mais il me semble qu'une des faiblesses de l'approche en terme de mythe tient justement à une tendance immanente au syncrétisme jusque sur le plan temporel, ce qui ne saurait satisfaire l'historien. En effet, même si l'auteur se défend d'essentialiser le " mythe chartrain ", il n'en a pas moins tendance à le considérer avant tout comme un flux quasi continu de récits se nourrissant eux-mêmes de leurs propres reprises, emprunts extérieurs et amplifications, au gré de "l'oubli et du hasard " (p. 235). Dans ce cadre, c'est le degré de " codification ", c'est-dire d'homogénéité et de cohérence des récits, qui vient structurer le séquençage historique et non les relations entre la fabrique légendaire et les processus sociaux, politiques et institutionnels qui affectent les milieux de production. Restaurer un peu plus de discontinuité entre les différentes phases de la fabrique légendaire avant la rupture du milieu du xvII ${ }^{\mathrm{e}}$ siècle permettrait sans doute de mieux comprendre l'articulation non seulement entre récits et pratiques sociales, mais aussi et surtout entre récits et enjeux socio-institutionnels. Bref, de réintroduire un peu de nécessité dans l'histoire.

Florian MAZEL

10. Iogna-Prat, Dominique, La Maison Dieu. Une histoire monumentale de l'Église au Moyen Âge, v. 800-v. 1200, Paris, 2006. 